\title{
Glioblastoma familiar múltiple de aparición metácrona: implicaciones etiopa- togénicas y pronósticas
}

\author{
B. Iza; O. Mateo-Sierra; F. Ruiz-Juretszke; J. Garbizu; J. Guzmán de Villoria* y R. Carrillo
}

Servicio de Neurocirugía, Servicio de Neurorradiología*, Hospital General Universitario Gregorio Marañón. Madrid.

\section{Resumen}

El Glioblastoma multiforme con agregación familiar es poco frecuente, asociándose la mayor parte de los casos a síndromes genéticos conocidos (como el síndrome de Turcot, el síndrome de Li-Fraumeni, la neurofibromatosis, etc). Sin embargo, existen otros gliomas familiares no asociados a estos cuadros sindrómicos que, aunque menos frecuentes, han mostrado unas características etiológicas y clínicas diferentes a las de los gliomas esporádicos.

Por otra parte, hasta un $\mathbf{1 0 \%}$ de los gliomas se consideran verdaderamente multicéntricos, apareciendo de modo síncrono o metácrono. Los glioblastomas de aparición metácrona han mostrado en algunos estudios un mejor pronóstico, habiéndose encontrado trastornos genéticos diferentes en los tumores de un mismo paciente.

Los gliomas familiares con presentación metácrona son excepcionales. Estos tumores presentan unas implicaciones terapéuticas especiales por la limitación del tratamiento radioterápico tras el tratamiento inicial. Aunque se han identificado mutaciones variadas en estos pacientes, la identificación precisa de dichos trastornos se basará en el estudio de su sustrato genético específico. Presentamos un caso clínico que combina ambas peculiaridades revisando las características de esta patología.

PALABRAS CLAVE: Glioma multicéntrico. Glioma familiar. Glioblastoma metácrono. Glioblastoma multiforme

Familiar glioblastoma presentig as a true multicentric tumor: etiopathogenic and pronostic features

\section{Summary}

Familial glioblastoma multiforme is a rather uncommon entity, being in most cases associated to known

Recibido: 6-09-05. Aceptado: 2-12-05 genetic disorders (as Turcot syndrome, Li-Fraumeni syndrome, neurofibromatosis, etc.). However, familial gliomas have also been described, although less frequently, independently of these genetic syndromes showing some special features regarding its etiology and clinical manifestations.

Less than $10 \%$ of gliomas may be considered as true multicentric tumours either synchronous or metachronous in clinical presentation. Metachronous glioblastomas have been associated to better prognosis in some studies, with genetic studies having found clear differences among the tumors within same patients.

Familial glioblastoma with metachronous presentation is an exceptional disorder. These tumors show special therapeutic implications due to the limitations of radiotherapy once the patient has already irradiated. A variety of non-specific mutations have been found in these patients but true characterization of this disorder remains unclear and will be based on further genetic studies. We present a clinical report on a patient harbouring a familial and metachronous glioblastoma. The main aspects of this entity are reviwed.

KEY WORDS: Multicentric glioma. Familiar glioma. Metachronous glioblastoma. Glioblastoma multiforme

Introducción

Los gliomas cerebrales son tumores preferentemente esporádicos. La agregación familiar de los mismos es muy poco frecuente y en general asociada a síndromes neurogenéticos bien descritos (síndromes de Turcot, de Li-Fraumeni, neurofibromatosis...). Sin embargo, existen casos de gliomas con agregación familiar que no se ajustan a síndromes conocidos, habiéndose descrito hasta una incidencia de gliomas diez veces superior en familiares de pacientes $^{14}$. Se han descrito características epidemiológicas y citogenéticas diferenciadas para los gliomas familiares, aunque aún no se conoce la base de la predisposición a esta patología.

Por otra parte, los gliomas múltiples, considerados 

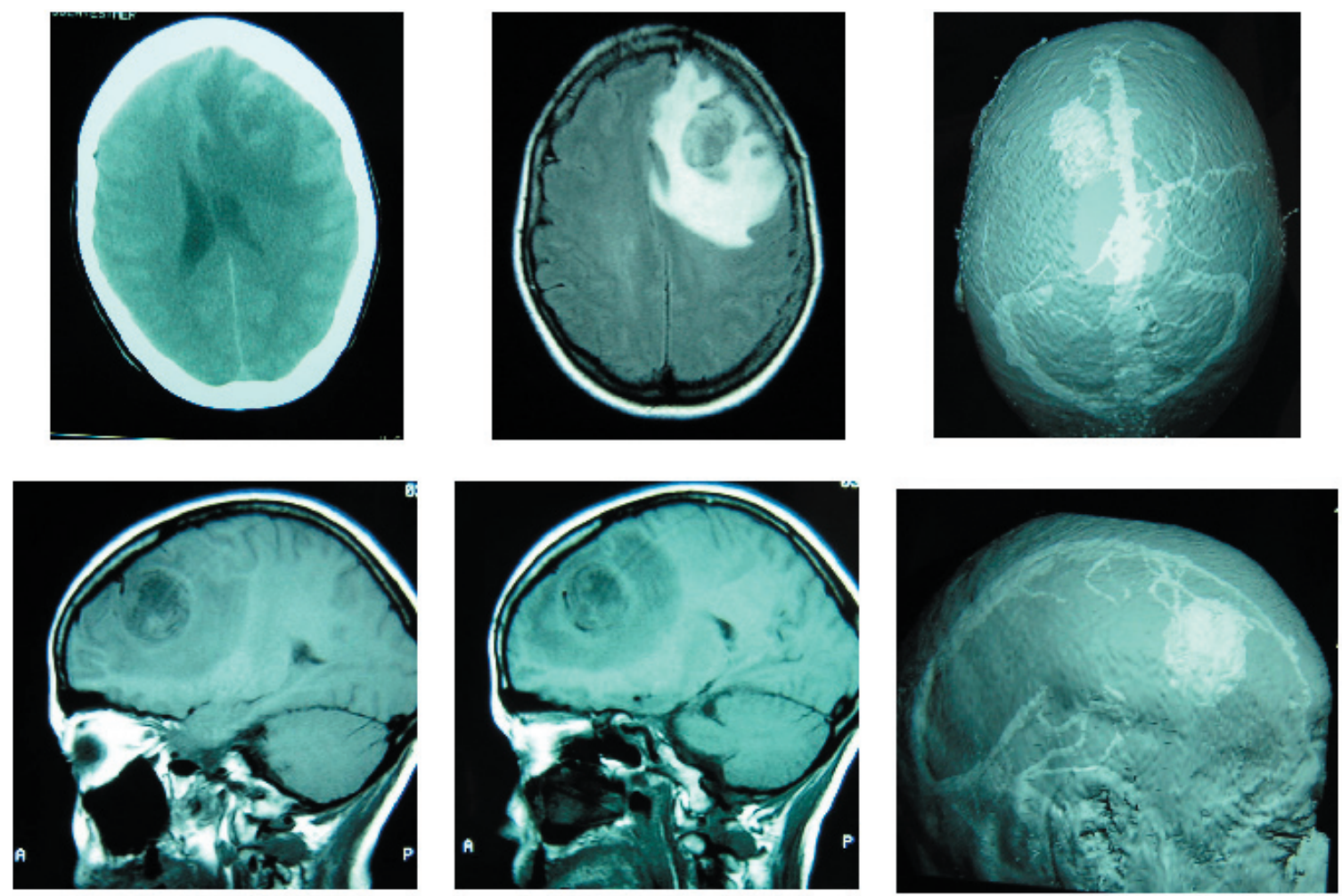

Figura 1. Imágenes de TC craneal (figura superior izquierda) y de RM craneal de la lesión inicial, tumor cortico-subcortical frontal izquierdo con importante edema vasogénico circundante.

como aquéllos con diferentes focos sin conexión macro ni microscópica, aparecen hasta en el $9 \%$ de los gliomas malignos?. Típicamente se trata de glioblastomas multiformes que pueden presentar una aparición síncrona o metácrona. También en esta variante de glioblastomas se han demostrado unas características clínicas y citogenéticas que permiten diferenciarlo del patrón habitual de comportamiento de los glioblastomas.

La asociación de glioblastomas multiformes familiares con un patrón múltiple de lesiones no es excepcional; se ha descrito una mayor frecuencia de evoluciones más benignas, con supervivencias más largas. Presentamos el caso clínico de una paciente con glioblastomas múltiples de presentación "metácrona", es decir no coincidente en el tiempo, con antecedentes familiares de tumores similares, que presenta un perfil evolutivo que se ajusta a las características de estas entidades descritas en la literatura.

\section{Caso clínico}

Paciente de 62 años de edad, con antecedentes personales de glaucoma crónico e histerectomía. Acude por un cuadro de pérdida de memoria de fijación, de aproximadamente un mes de evolución, inicialmente etiquetado como síntoma de un proceso depresivo. No presenta focalidad neurológica a la exploración física. Como antecedentes familiares destaca que tanto su madre como su abuela fallecieron por un glioblastoma cerebral. En la tomografía computarizada (TC) craneal se evidencia la presencia de un proceso expansivo intraaxial córtico-subcortical frontal izquierdo; los hallazgos de imagen en resonancia magnética (RM) de dicha lesión se consideran compatibles con un glioma de alto grado, localizado en región hemisférico premotora izquierda. (Figura 1).

Inicialmente se realiza tratamiento quirúrgico mediante una craneotomía frontal izquierda, con resección completa del tumor, cuyo informe histológico confirma el diagnóstico de glioblastoma multiforme. En la TC de control del primer día de postoperatorio se confirma la ausencia de restos tumorales (Figura 2). Posteriormente se somete a tratamiento coadyuvante con radio y quimioterapia, según los protocolos habituales, sin morbilidad derivada de ninguno de estos tratamientos.

La evolución de la paciente es favorable, permaneciendo asintomática en los sucesivos controles. A los 13 meses de la cirugía se detecta en una RM de control una lesión cortico-subcortical frontal derecha sugestiva de glioma de alto grado, sin aparente conexión con el tumor contralateral intervenido y sin signos de recidiva de dicha lesión intervenida (Figura 3). Es reoperada a los 14 meses 

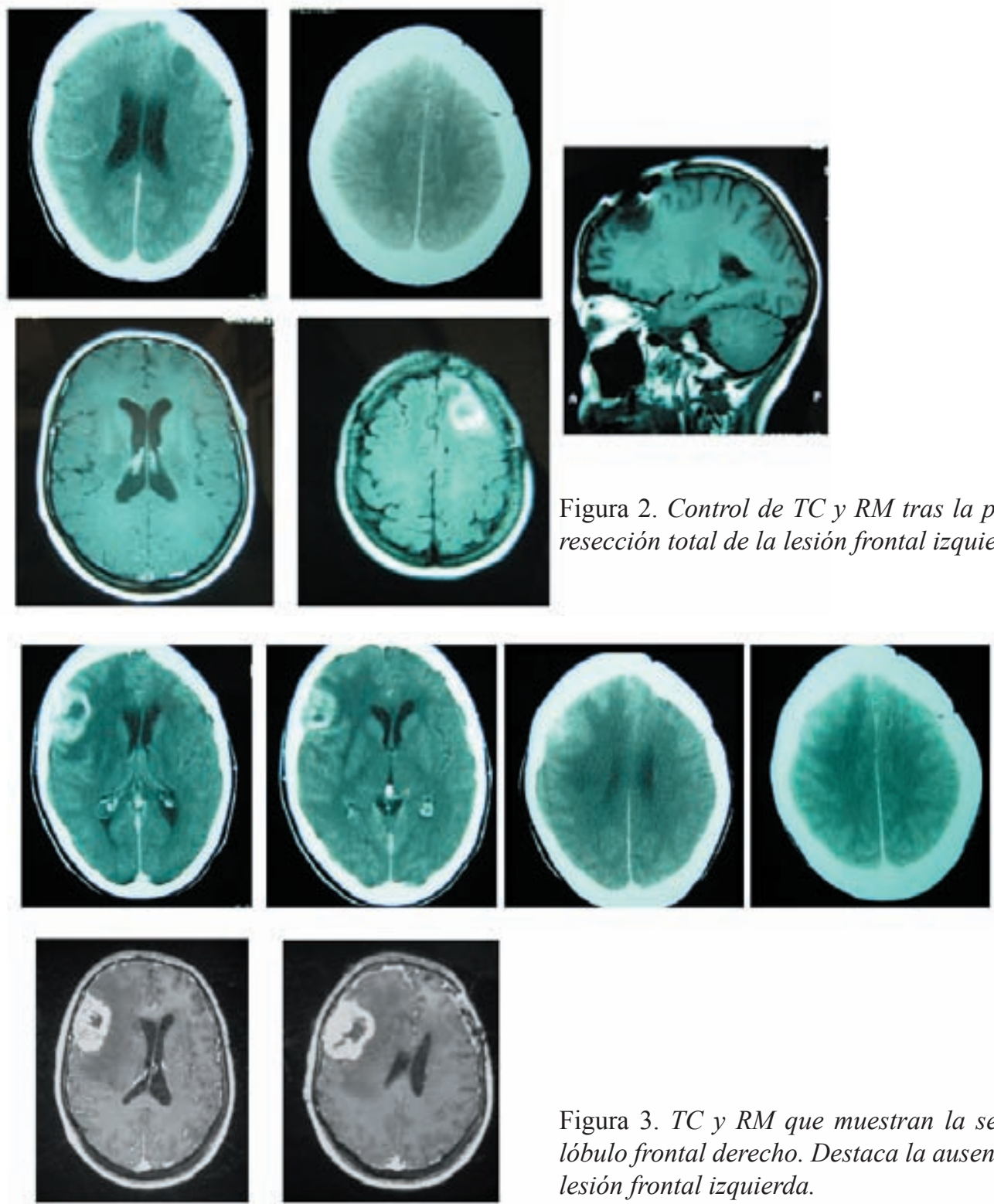

Figura 2. Control de TC y RM tras la primera cirugía, que muestra resección total de la lesión frontal izquierda.

Figura 3. TC y RM que muestran la segunda lesión, localizada en lóbulo frontal derecho. Destaca la ausencia de recidiva de la primera lesión frontal izquierda.

de la primera cirugía mediante craneotomía frontal derecha con resección completa del tumor (Figura 4). El informe histológico confirma que es un glioblastoma. En la TC de control postoperatoria se verifica la resección completa de la lesión derecha (Figura 5). Valorada por Oncología se descarta radioterapia coadyuvante y se decide tratamiento con quimioterapia.

La paciente persiste asintomática. A los tres meses de la segunda cirugía y en una TAC de control se apreció un nódulo de pequeño tamaño temporal basal derecho que capta contraste y es sugestivo de un nuevo foco de glioblastoma (Figura 6). La paciente rechaza tratamientos invasivos y se somete a tratamiento conservador paliativo. En posteriores estudios de imagen se objetivan nuevas lesiones tumorales. La paciente fallece 26 meses después de la primera cirugía.

\section{Discusión}

\section{Gliomas familiares}

Aunque la gran mayoría de los tumores cerebrales, especialmente los gliomas, se presentan de forma esporádica, algunos estudios epidemiológicos demuestran ciertos casos de agregación familiar ${ }^{5}$. Muchos son consecuencia de desórdenes neurogenéticos o multisistémicos bien descritos (Von Hippel Lindau, neurofibromatosis, Turcot, Li-Fraumeni, esclerosis tuberosa), si bien una pequeña pro- 

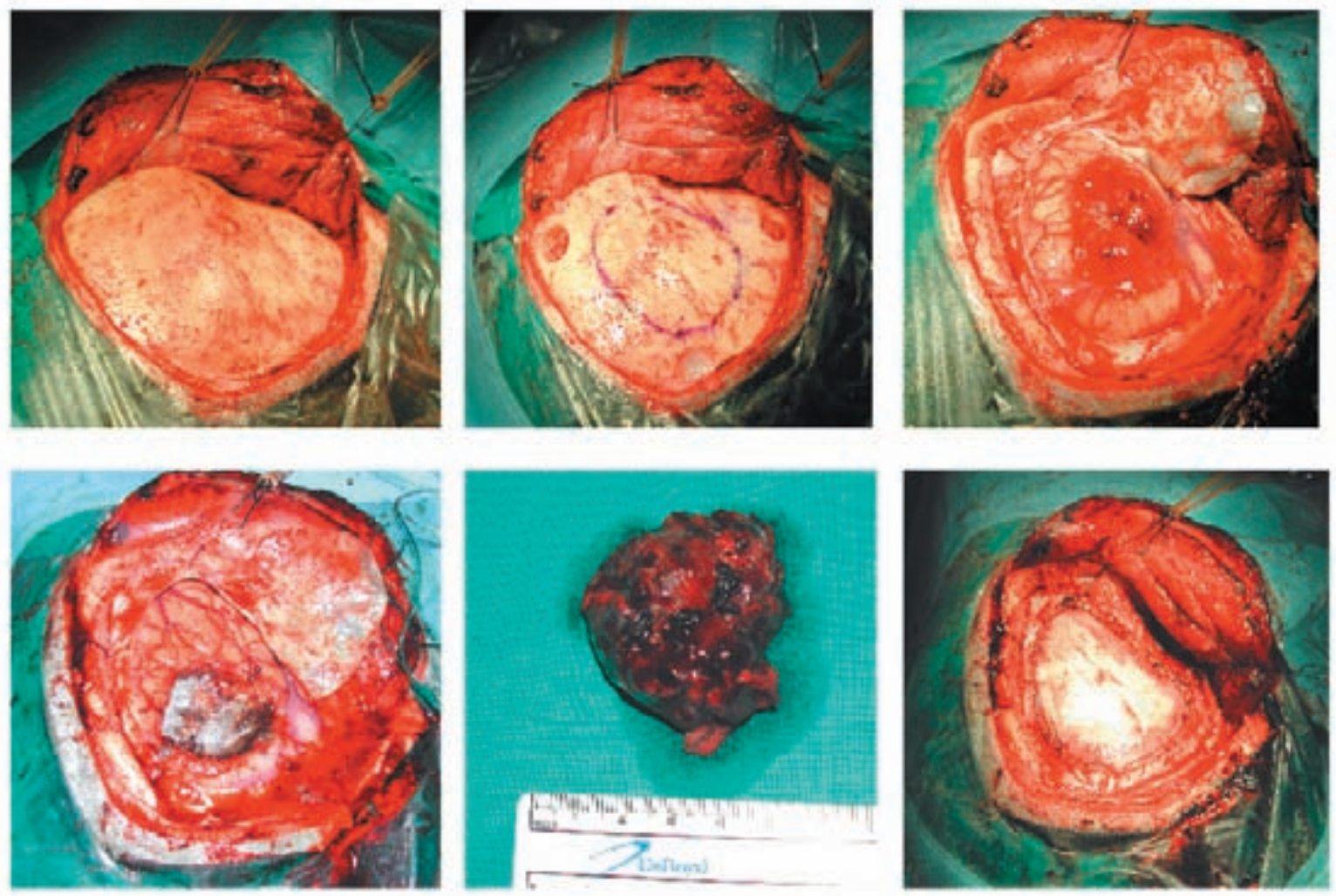

Figura 4. Imágenes correspondientes a la segunda cirugía, con extirpación completa de la lesión frontal derecha.
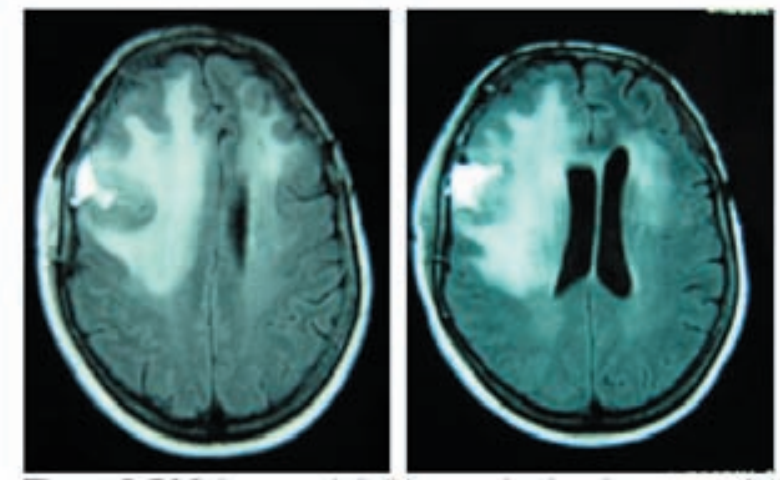

Figura 5. RM de control de la segunda cirugía, se aprecia la resección completa.

porción no se ajusta a ningún síndrome hereditario conocido. Característicamente, estos últimos afectan a pacientes más jóvenes ${ }^{8}$, a distintas generaciones de diferente procedencia geográfica y no se presentan en cónyuges, datos que apoyan un origen genético más que debido a factores ambientales.

Se ha calculado la incidencia de la agrupación familiar de los gliomas, independientemente de cuadros sindrómicos conocidos, por diversos estudios que arrojan resultados no determinantes, aunque sí llegan a demostrar que dicha agregación existe y que no es fruto del azar
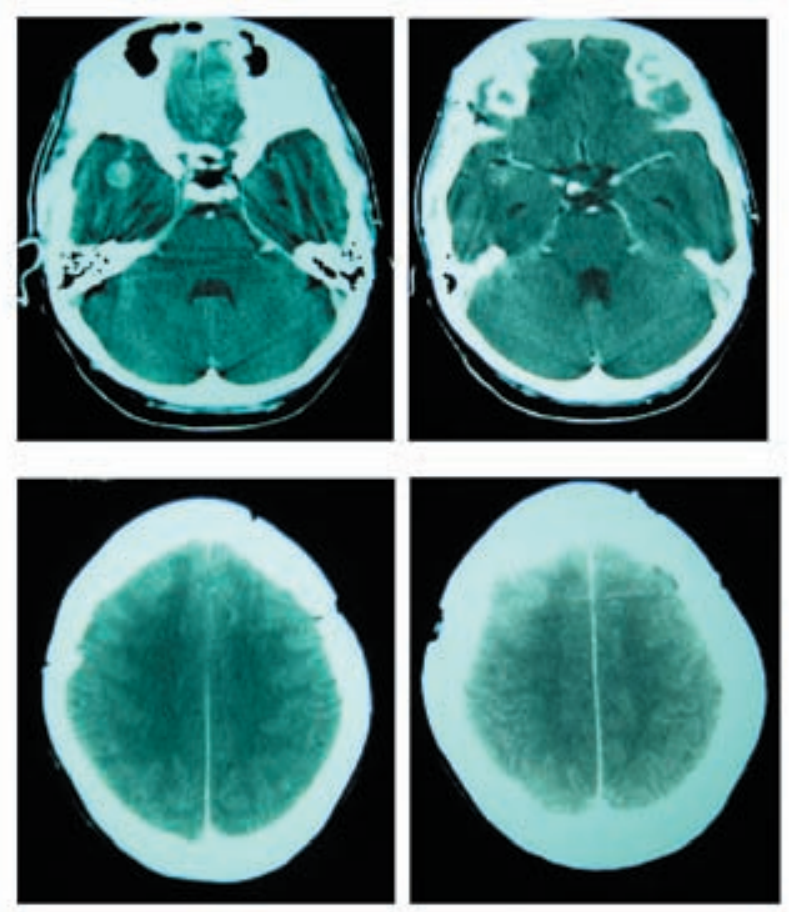

Figura 6. TC en el que se evidencia la aparición de una tercera lesión, en este caso afectando a la punta del lóbulo temporal derecho. Ausencia de recidiva de los otros dos focos tumorales.. 


\begin{tabular}{|c|l|}
\hline \multicolumn{2}{|c|}{ Datos de estudios epidemiológicos sobre agregación familiar de gliomas } \\
\hline Autor & \\
\hline Van de Weil y cols., $1960^{24}$ & $\begin{array}{l}\text { Mortalidad por glioma x } 4 \text { en familiares de paciente con glioma } \\
\text { Incidencia x 10 en familiares primer grado vs controles } \\
\text { Metzel y cols,.1964 }\end{array}$ \\
Lossignol y cols., $1990^{11}$ & $9,4 \%$ de pacientes con glioma tienen familiar de primer grado afecto \\
\hline
\end{tabular}

\begin{tabular}{|c|c|}
\hline \multicolumn{2}{|r|}{$\begin{array}{c}\text { Tabla } 2 \\
\text { Incidencia de gliomas múltiples según diferentes series }\end{array}$} \\
\hline Autor & Incidencia gliomas múltiples \\
\hline Russel y cols., $1989^{19}$ & $2-10 \%$ de necropsias \\
\hline Van Tassel y cols., $1988^{23}$ & En $5 \%$ de TC de pacientes con glioma (2,5\% síncronas , 2,5\% metácronas) \\
\hline Djalilian y cols., $1995^{7}$ & El 9\% de pacientes con glioma presentan otra lesión en ese momento o posteriormente \\
\hline
\end{tabular}

(Tabla 1) . Lossignoll ${ }^{11}$ y cols., calculan en su serie que un $9.4 \%$ de los pacientes con astrocitomas anaplásicos tienen al menos un familiar de primer grado con un tumor de estirpe astrocítica; Metzel ${ }^{14}$ estima una incidencia de gliomas en familiares de enfermos diez veces superior a la de los controles. Van der Wei $1^{24}$ concluye que los familiares de pacientes con glioma tienen una mortalidad por dicho tumor cuatro veces superior a la de los controles.

La evidencia definitiva pasa por la identificación molecular genética de esta predisposición, aunque no existen suficientes datos actualmente. Sí que se han observado ciertas alteraciones citogenéticas, como las delecciones en los brazos largos de los cromosomas 4 y 6 , o bien las ganancias 1 p y $22 \mathrm{q}$, frecuentemente asociadas a los gliomas familiares no asociados a síndromes hereditarios ${ }^{17}$. Aunque en el caso aquí presentado es evidente la agregación familiar, no se pudieron realizar estudios genéticos para compararlos con los hallados de series previas publicadas.

De un modo más especifico, desde el punto de vista de la genética molecular, se ha estudiado la presencia de mutaciones en el gen p53 en estos pacientes. Se sabe que la mutación somática forma parte de los eventos genéticos precoces en la histogénesis de los gliomas esporádicos y que no se pierde en la evolución del mismo (ni en el cultivo "in vivo" ni en la dispersión metastásica del mismo).

La alta frecuencia de gliomas en pacientes con síndrome de Li-Fraumeni, causado por mutación de p53 en células germinales, y la presencia de mutaciones somáticas de p53 en los gliomas esporádicos, arrojaba la posibilidad de que esta alteración en células germinales tuviese algún papel en la histogénesis tumoral en familias con gliomas no asociados al síndrome de Li-Fraumeni. En el estudio genético de 18 familias con glioma no afectadas por dicho síndrome, Paunu ${ }^{17}$ no encuentra en ninguna de ellas esta alteración en células germinales; es más, concluye que la incidencia de mutación de p53 en células somáticas es similar en pacientes con glioma familiar y en aquéllos con esporádico ${ }^{17}$. Por tanto, no parece que el sustrato genético de la agregación familiar de los gliomas resida en el gen p53 y se requerirán futuros estudios de citogenética para identificar dicha predisposición.

\section{Gliomas múltiples metácronos}

El primer registro de lesión gliomatosa múltiple lo realiza Bradley ${ }^{2}$ en 1880 . Los estudios de necropsia calculan una incidencia de $2-10 \%$ de multicentricidad de lesiones gliomatosas sin conexión macro o microscópica ${ }^{3,19}$ y ya es conocida la predisposición de pacientes con esclerosis mútiple ${ }^{10}$ o síndrome de Von Recklinghausen ${ }^{19,21}$ al desarrollo de gliomas múltiples. Van Tassel ${ }^{23}$ recoge una serie de 21 casos de lesiones múltiples (11 síncronas y 10 metácronas) entre 440 pacientes afectados de glioma, y estima una incidencia radiológica en $\mathrm{TC}$ de un 5\%. Un estudio más reciente, que incluye TC pero también RM de 100 pacientes consecutivos con glioma maligno, concluye que el $9 \%$ de los pacientes con un glioma maligno presentan otra lesión, bien sea en ese mismo momento o desarrollada posteriormente (Tabla 2). Habitualmente se trata de lesiones supratentoriales, aunque con la generalización de la RM, cada vez se identifican más lesiones en la fosa posterior. El subtipo histológico más frecuentemente encontrado en ambas lesiones es el glioblastoma multiforme ${ }^{6,20,21}$, si bien se han registrado casos de astrocitomas de bajo grado y de 
ependimomas ${ }^{10,15}$.

Existen varias teorías etiopatogénicas al respecto: según Willis ${ }^{26}$ existe una primera fase de transformación neoplásica de una gran área de tejido cerebral, que posteriormente desarrolla lesiones distantes independientes por las distintas tasas de mitosis. Según Zülch ${ }^{27}$ se trataría de metástasis de un foco primario, sin quedar clara la vía de diseminación.

En cuanto a las lesiones metácronas, separadas en el tiempo con confirmación radiológica, suponen un $6 \%$ de la serie de Djalilian ${ }^{7}$. La vía de diseminación por LCR, que supondría la segunda lesión como siembra tardía de la primera, es improbable. El papel etiopatogénico de la radioterapia empleada como tratamiento de la lesión inicial queda prácticamente descartado dado el escaso lapso de tiempo que suele separar ambas lesiones, insuficiente para el desarrollo de la acción oncogénica de la radiación. En la mayoría de los casos el análisis histológico de la segunda lesión descarta la existencia de radionecrosis. Además se ha planteado también la posibilidad de la migración de células malignas de la lesión inicial a través de la sustancia blanca y su siembra dando lugar eventualmente a la segunda lesión, pero actualmente se desconoce la etiología de estas lesiones. En la paciente aquí presentada no parece clara ninguna hipótesis etiopatogénica aparte de la secundaria a la posible predisposición genética.

Genéticamente se trata de procesos malignos independientes y consecutivos con patrones moleculares diferentes $^{13}$. Presentan anomalías propias de glioblastomas tanto primarios (mutaciones en gen PTEN, delección p16) como secundarios (mutaciones p53, ausencia de amplificación EGFR $)^{18}$, con lo que podría establecerse una tercera categoría de glioblastomas, que encajaría con ese $30 \%$ de ellos que no coinciden en ninguno de esos subgrupos ${ }^{12,25}$. La aparición de lesiones separadas en el tiempo es un indicador de inestabilidad genética celular, bien cromosómica o de microsatélites o a nivel de nucleótidos (estas dos no confirmadas en el estudio de Reis ${ }^{18}$ ).

\section{Valor pronóstico. Implicaciones terapéuticas}

En general tanto los gliomas con agregación familiar como los de presentación metácrona múltiple conllevan un pronóstico algo mejor que los solitarios y esporádicos, por lo que se encuentra justificada una mayor agresividad en el enfoque terapéutico; Reis ${ }^{18}$ ha registrado supervivencias de 10 años entre la primera y segunda lesión, y Martínez y $\operatorname{cols}^{13}$ mostraron una supervivencia de 5 años en su caso. Tal vez se corresponda con el subgrupo de glioblastomas (el $2 \%$ del total ${ }^{22}$ ) que presentan una supervivencia mayor $\mathrm{o}$ igual a 3 años.

Respecto a las causas de este mejor pronóstico se han encontrado datos similares al de estos gliomas en tumores de otros órganos. Se ha demostrado que los cánceres colorrectales y gástricos que se desarrollan como consecuencia de inestabilidad de microsatélites, presentan un mejor pronóstico ${ }^{9,16}$. Se ha sugerido que el cúmulo de tantas alteraciones genéticas conlleve la apoptosis precoz de las células malignas ${ }^{4}$, controlándose así, hasta cierto punto, la tasa de crecimiento tumoral. Otra posibilidad descrita es que ciertas mutaciones conduzcan a la presentación de antígenos de superficie nuevos que desencadenen una respuesta inmunoprotectora $^{1,9}$.

Además, se ha determinado una mutación específica en tres pacientes con glioma metácrono que han mostrado una supervivencia larga: 6 de 7 mutaciones puntuales de p53 y PTEN en los dos tumores del caso de Reis y $\operatorname{cols}^{18} \mathrm{y}$ en uno de los de Martínez y $\operatorname{cols}^{13}$ son consecuencia de desaminación de 5-metilcitosina. Evidentemente, es necesario el estudio de más muestras antes de suponer que esta mutación está relacionada con lesiones de mejor supervivencia. En la paciente presentada no sólo es llamativo el patrón temporal de aparición de las nuevas lesiones sino también la ausencia de recidiva local a los dos años de la primera cirugía, lo que es muy infrecuente en este tipo de tumores. Desde el punto de vista de tratamiento adyuvante a la cirugía, la aparición metácrona de estos tumores plantea la limitación de la radioterapia, ya utilizada en el primer episodio gliomatoso, por lo que se recomienda la utilización de técnicas de radiocirugía para minimizar los efectos deletéreos de la radiación sobre un campo que ya ha sido irradiado hace relativamente poco tiempo ${ }^{5,7}$. Sin embargo no existe variación en los regímenes quimioterápicos empleados tanto en primera como en segunda línea.

\section{Conclusión}

Tanto la existencia de lesiones gliomatosas independientes y separadas en el tiempo como la agrupación familiar de gliomas constituyen hechos aislados y excepcionales, teniendo en cuenta la frecuencia de este tipo de neoplasias cerebrales. Los escasos estudios publicados al respecto muestran una posible tendencia de estos tumores a presentar supervivencias más largas que las de los gliomas esporádicos aislados. En cualquier caso, parece que la identificación del origen de la multicentricidad de los gliomas o de su predisposición familiar se basará en estudios de genética molecular que permitirán, a su vez, filiar estos trastornos y probablemente identificar los pacientes con mejor pronóstico.

\section{Bibliografía}

1. Bodmer, W., Bishop, T., Karran, P.: Genetic steps in colorectal cancer. Nat Genet 1994; 6: 217-219.

2. Bradley, W.L.: Case of gliosarcomatous tumors of the 
brain. Proc Conn Med Soc 1880; 2: 39-41.

3. Bussone, G., Sinatra, M.G., Boiardi, A., Lazzaroni, M., Mariani, C., Allegranza, A.: A case of glioblastoma with múltiple centres above and below the tentorium. J Neurol 1979; 221: 187-192.

4. Cahill, D.P., Kinzler, K.W., Vogelstein, B., Lengauer, C.: Genetic instability ad Darwinian selection in tumors. Trends Cell Biol 1999; 9: M59-M60.

5. Caroli, E., Salvati, M., Peruzzi, P., Frati, A., Giangaspero, F. : Familial gliomas. Analysis of six families with gliomas and without other inheritable syndromes. Neurosurg Rev 2003; 26: 280-282.

6. Chadduch, W.M., Roycroft, D., Brown, M.W.: Multicentric glioma as a cause of multiple cerebral lesions. Neurosurg 1983; 13: 170-175.

7. Djalilian, H.R., Shah, W.V., Hall, W.A.: Radiographic incidence of multicentric malignant gliomas. Surg Neurol 1995; 51: 554-558.

8. Grossman, S.A., Osman, M., Hrubam, R., Piantadosi, S.: Central nervous system cancers in first-degree relatives and spouses. Cancer Inv 1999; 17: 299-308.

9. Kim, H., Jen., J., Voglestein, B., Hamilton, S.R. : Clinical and pathological characteristics of sporadic colorectal carcinomas with DNA replication errors in microsatellite sequences. Ann J Pathol 1994; 145: 148-156.

10. Kotwica, Z., Papierz, W.: Cerebral and cerebellar glial tumors in the same individual. Neurosurg 1992; 30: 439-442.

11. Lossignol, D., Grossman, S.A., Sheider, V.R., Griffin, C.A., Piantadosi, S.: Familiar clustering of malignant astrocytomas. J Neurooncol 1990; 9: 139-145.

12. Louis, D.N.: A molecular genetic model of astrocytoma histopathology. Brain Pathol 1997; 7: 755-764.

13. Martínez, R., Schackert, H-K, Von Kannern, S., Lichter, P., Joos, S., Schackert, G.: Independent molecular development of metachronous glioblastomas whit extended intervening recurrence-free interval. Brain Pathol 2003; 13: 598-607.

14. Metzel, E.: Betrachtungen zur Genetik der Familiaren Gliome. Acta Genet Med Gemellol 1964; 13: 124-131.

15. Moertel, C.G., Dockerty, M.B., Baggenstoss, A.H.: Multiple primaty malignant neoplasm. III. Tumors of multicentric origin. Cancer 1961; 14: 238-248.

\section{Comentario al trabajo Glioblastoma familiar múltiple de aparición metácrona: implicaciones etiopatogénicas y pronósticas de $\mathrm{B}$. Iza y cols}

Iza y cols., presentan el caso de una paciente de 62 años diagnosticada de un glioblastoma multiforme con dos peculiaridades inusuales. En primer lugar, la paciente presentaba antecedentes familiares de glioblastoma multiforme (madre
16. Papadopoulus, N., Nicolaides, N.C., Wei, Y.F., et al.: Mutation of a multiL homolog in hereditary colon cancer. Science 1994; 263: 1625-1629.

17. Paunu, N., Syrjakosji, K., Sankila, R., et al.: Analysis of p53 tumor suppresor gene in families with multiple glioma patients. J Neurooncol 2002; 55: 159-165.

18. Reis, R.M., Herva, R., Brandners, S., et al.: Second Primary glioblastoma. J Neuropathol Exp Neurol 2001; 60 : 208-215.

19. Russel, D.S., Rubenstein, L.J.: Pathology of tumors of the nervous system. Baltimore; Williams \& Wilkins, 1989; pp 124-126.

20. Sherer, H.J.: The forms of growth in glioma and their practical significance. Brain 1940; 63: 1-35.

21. Solomon, A., Perret, G.E., McCormich, W.F.: Multicentric gliomas of the cerebral and cerebellar hemispheres. Case report. J Neurosurg 1969; 31: 887-893.

22. Scott, J.N., Rewcastle, N.B., Brasher, P.M.A., et al.: Which glioblastoma multiforme patient will become a longterm survivor? A population based study. Ann Neurol 1999; 46: 183-188.

23. Van Tassel, T., Lee, Y., Burner, J.M.: Synchronous and metrachronous malignant gliomas. CT findings. AJNR 1988; 9: 725-732.

24. Van de Weil, H.J.: Inheritance of glioma. New York, Elsevier; 1960.

25. Von Deimling, A., Louis, D.N., Wiestler, O.D.: Molecular pathways in the formation of gliomas. Glia 1995; 15: 328-338.

26. Willis, R.A.: Pathology of tumors. London; Butterworths, 1960: pp 811.

27. Zülch, K.J.: Brain tumors. Their biology and pathology. New York; Springer, 1957: pp 74-6.

Iza, B.; Mateo-Sierra, O.; Ruiz-Juretszke, F.; Garbizu, J.; Guzmán de Villoria, J.; Carrillo, R.: Glioblastoma familiar múltiple de aparición metácrona: implicaciones etiopatogénicas y pronósticas. Neurocirugía 2006; 17: 340-347.

Correspondencia postal: Dra. Begoña Iza. Servicio de Neurocirugía. Hospital General Universitario Gregorio Marañón. c/ Doctor Esquerdo 46. 28007 Madrid

y abuela). En segundo lugar, la enfermedad se manifiesta de manera multicéntrica y metácrona. La paciente recibe tratamiento quirúrgico en dos ocasiones, coadyuvancia con radio y quimioterapia tras la primera intervención y sólo 
quimioterapia tras la segunda, siguiendo los estándares de tratamiento actuales para esta patología. La supervivencia de 26 meses supera la mediana esperada para una paciente de 62 años con un glioblastoma multiforme.

El incremento de riesgo para desarrollar tumores cerebrales primarios en familiares de primer grado de pacientes afectos de meningiomas o gliomas (al margen de los síndromes hereditarios) ha sido demostrado en estudios epidemiológicos ${ }^{2}$. La ausencia de un incremento de riesgo en los cónyuges de los mismos y el hecho de que hasta el $60 \%$ de los familiares afectos tengan edades inferiores a los 40 años en el momento del debut de la enfermedad, apunta más hacia un componente genético de predisposición que hacia un origen en la exposición ambiental a factores oncogénicos. Sin embargo, los estudios realizados hasta la fecha, no han podido demostrar de forma clara las alteraciones genéticas implicadas en esta predisposición familiar. Así, la expresión aberrante del gen supresor p53 se ha mostrado tan común en los casos de presentación esporádica como en los de agrupación familiar. Del mismo modo, la disfunción de genes implicados en la reparación del DNA no parece estar incrementada en los casos de gliomas familiares.

Los gliomas multicéntricos se definen por la presencia de lesiones separadas ampliamente en diferentes lóbulos o incluso en diferentes hemisferios cerebrales, y que a diferencia de los gliomas multifocales, no resultan de la diseminación de las células tumorales a través de comisuras, vías o del líquido cefalorraquídeo. La presentación de dichas lesiones, puede ocurrir separada en el tiempo, tal y como sucede en el caso descrito por Iza y cols. Se producen con mayor frecuencia en el compartimento supratentorial y el glioblastoma multiforme es el subtipo histológico más frecuente entre los gliomas multicéntricos ${ }^{3}$. La hipótesis más aceptada para explicar esta multicentricidad sugiere que las lesiones resultarían de un proceso en dos fases ${ }^{1}$ : una primera de "iniciación" donde una gran área o incluso todo el cerebro adquieren una mayor susceptibilidad para el desarrollo neoplásico, y un segundo paso de "promoción" que consistiría en una proliferación neoplásica en distintos puntos estimulada por factores bioquímicos, hormonales, mecánicos o virales.

El avance en el conocimiento de la genética molecular, ha permitido en los últimos años identificar subgrupos de pacientes con tumores histopatológicamente similares, y sin embargo con un comportamiento clínico, de respuesta al tratamiento y por tanto un pronóstico diferentes. Todo ello nos permitirá en un futuro, esperemos no muy lejano, ofrecer un tratamiento mucho más individualizado y probablemente más eficaz a los pacientes "clásicamente" incluidos bajo un único epígrafe nosológico como el de glioblastoma multiforme.

\section{Bibliografía}

1. Knudson, A.G.: Two genetic hits (more or less) to cancer. Nat Rev Cancer 2001; 1: 157-162.

2. Malmer, B., Henriksson, R., Grónberg, H.: Familial brain tumours-genetics or environment? A Nationwide cohort study of cancer risk in spouses and First-degree relatives of brain tumour patients. Int J Cancer 2003; 106: 260-263.

3. Salvati, M., Caroli, E., Orlando, E.R, et al.: Multicentric glioma: our experience in 25 patients and critical review of the literature. Neurosurg Rev 2003; 26: 275279.

M. Brell

Palma de Mallorca 